\title{
ANÁLISIS FITOLÍTICO DEL SITIO ARQUELÓGICO YASYAMAYO (SANTA MARÍA - TUCUMÁN - ARGENTINA): PROCESOS ANTRÓPICOS Y PALEOAMBIENTALES
}

\author{
PHYTOLITHIC ANALYSIS OF THE YASYAMAYO ARCHAEOLOGICAL SITE \\ (SANTA MARÍA - TUCUMÁN - ARGENTINA): ANTHROPOGENIC AND \\ PALEOENVIRONMENTAL PROCESSES
}

\author{
María Gisela Lefebvre', María de los Milagros Colobig², Alejandro Fabián Zucol², Mario Gabriel \\ Maldonado y María Marta Sampietro Vattuone ${ }^{1}$
}

\begin{abstract}
Se dan a conocer los resultados de la descripción de la población de microrrestos vegetales recuperados de perfiles localizados en terrazas de cultivo de la localidad de Yasyamayo (Valle de Santa María, Tucumán, Argentina), pertenecientes al periodo de Desarrollos Regionales (ca. 1000-600 AP). Los fitolitos fueron extraídos del sedimento mediante el procesamiento físico y químico de las muestras. Los resultados mostraron que las asociaciones fitolíticas en los perfiles antropizados se caracterizan por poseer morfotipos principalmente de origen graminoide (prismáticos, conos truncados, aguzados, flabelados, bilobados), y por la presencia de elementos afines a las Maideas en los niveles de ocupación humana. El aporte de elementos panicoides y los indicadores de condiciones húmedas (como las diatomeas) en estos niveles de ocupación darían cuenta de las estrategias de manejo empleadas por los grupos humanos de esta zona en el pasado. Este trabajo constituye la primera aproximación al objeto de estudio en el área. Por otra parte, la concentración de diatomeas de modo diferencial permite estimar la utilización de técnicas de regadío por los grupos humanos que habitaron en dicho sitio.
\end{abstract}

Palabras claves: Yasyamayo, paleoambiente, fitolitos, agricultura prehispánica, Holoceno superior.

This paper presents the results obtained from the analysis of the assemblage of plant microremains recovered from profiles in agricultural terraces in the archaeological site of Yasyamayo (Santa María Valley, Tucumán, Argentina) and which correspond to the Regional Developments Period (ca. 1000-600 BP). The phytoliths were extracted by physical and chemical processing of the samples. The results showed that the phytolith assemblages in the anthropogenic profiles are characterized by having morphotypes of mainly graminoid origin (prismatic, truncated, sharp, flabellum, and bilobed cones) and by the presence of Maideas on the human occupation levels. The presence of panicoid elements and the evidence of higher humidity levels (such as the diatoms) on these levels of occupation would account for the management strategies used by the human groups of this area in the past. This paper constitutes the first approach to the phytolith study in the area. On the other hand, the differential concentration of diatoms enables the estimation of irrigation techniques by the human groups that inhabited this site.

Key words: Yasyamayo, paleoenvironment, phytoliths, prehispanic agriculture, Upper Holocene.

Un fitolito es un cuerpo mineralizado de origen vegetal (Bertoldi de Pomar 1971; Zucol 1992), integrante de tejidos orgánicos producidos por sustancias ergásticas. Con la muerte del vegetal o de alguno de sus órganos, los tejidos se incorporan al sustrato y luego de la degradación de los componentes orgánicos, los fitolitos son liberados (Baker 1960; Wilding 1967). Estos son altamente resistentes y tienen baja tasa de deterioro en los sedimentos arqueológicos, lo que permite la identificación de vegetales. Se conservan en estructuras de combustión, en cuencos utilizados para la cocción de alimentos, entre otros (Zurro 2006). Su gran resistencia también les permite perdurar en secuencias estratigráficas del Cuaternario (Capdepont et al. 2005; Piperno y Jones 2003; Piperno et al. 2007). Esta propiedad resulta de gran importancia para el estudio de las prácticas agrícolas llevadas a cabo por sociedades pasadas, y cómo estas afectaron el paisaje a lo largo del tiempo, es decir, cómo se han manejado ciertos cultivos o propiciado el crecimiento y distribución de

\footnotetext{
${ }^{1}$ Laboratorio de Geoarqueología. Facultad de Ciencias Naturales e Instituto Miguel Lillo. UNT. CONICET. Av. Alem 114, 4000, Tucumán, Argentina. gisela_lefebvre_2005@hotmail.com; gabrielmaldonado23@yahoo.com.ar; sampietro@tucbbs.com.ar

${ }^{2}$ Centro de Investigaciones Científicas y Transferencia de Tecnología a la Producción (CICYTTP-UADER-Provincia de Entre RíosCONICET), Dr. Materi y España SN, E3105BWA, Diamante, Argentina. milagroscolobig@ gmail.com; cidzucol@gmail.com
} 
determinados grupos vegetales muy vinculados a la actividad humana. Además, pueden servir para conocer la interrelación de los silicofitolitos con la cobertura vegetal conocida, la pedogénesis de suelos y las características paleoambientales de sitios arqueológicos (Borrelli et al. 2008; Fernández Honaine et al. 2009; Gallego et al. 2004; Iriarte y Alonso Paz 2009; Pearsall 2000). Los silicofitolitos son especialmente abundantes en las familias de las gramíneas y de las ciperáceas. La clasificación de Twiss et al. (1969) ha sido ampliamente utilizada por los investigadores y readaptada en varias ocasiones y la última modificación fue realizada en 1992 (Twiss 1992) con la separación de tres subfamilias dentro de las gramíneas: festucoides, chloridoides y panicoides, debido a la información de tipo climático que proporcionan cada una de ellas.

En Argentina y Chile hacia ca. 500 AP, fueron descritos fitolitos de especies vegetales domesticadas como el maíz, batata, poroto, quínoa y calabaza y si bien esta información aún es escasa y fragmentaria hacia la zona central de Chile, permite señalar que las plantas fueron parte del universo ideacional y cultural participando activamente en la configuración de los modos de vida e identidades sociales. (Pearsall 2008; Planella et al. 2014).

En el Noroeste Argentino, los estudios de microfósiles se aplicaron en algunos sitios arqueológicos con el objeto principal de determinar las especies cultivadas localmente y reconstruir las prácticas agrarias pasadas mediante el análisis de muestras de sedimento extraídas de estructuras agrícolas. Tal es el caso de los trabajos realizados en el Valle de El Bolsón (Departamento Belén, Catamarca) por Korstanje (2005, 2009). Allí, Korstanje y Cuenya (2008) identificaron microfósiles vegetales pertenecientes a las familias Chenopodiaceae, Solanaceae, Cucurbitaceae y Basellaceae en sedimentos extraídos de terrazas agrícolas. En las terrazas agrícolas con dos paredes laterales encontraron una gran cantidad de almidones circulares medianos del tipo Zea mays (maíz). En el mismo valle, en la localidad de Alto Juan Pablo, Maloberti (2014) extrajo muestras de sedimentos procedentes de estructuras agrícolas e identificó fitolitos pertenecientes a las subfamilias Pooideae, Panicoideae y Arundinoideae.

Williams et al. (2010) analizaron muestras de sedimentos de terrazas agrícolas del Valle Calchaquí Medio, y destacaron la muy baja frecuencia de silicofitolitos (excepto fitolitos de Poaceae), ausencia de almidones de maíz y la presencia de gránulos de almidón de tubérculos (afines a Solanum tuberosum) muy frecuente en algunas muestras. Zucol et al. (2012) realizaron el estudio de distintos tipos de microfósiles vegetales en la zona de Ambato (Los Varela, provincia de Catamarca, Argentina), como así también del ambiente de desarrollo de las culturas locales. Las asociaciones de microrrestos estuvieron mayoritariamente compuestas por fitolitos, con un marcado predominio de los fitolitos unicelulares (disociados en distintos tipos de sustratos). Los fitolitos observados son principalmente de gramíneas con escasa presencia de ciperoides, arecoides y de afinidad dicotiledónea. En forma complementaria, Zucol et al. (2015) establecen una mayor abundancia de fitolitos megatérmicos (tanto panicoides como chloridoide) sobre los microtérmicos (pooides y estipoides) y mesotérmicos (danthonioides). Además, constatan la presencia de granos de almidón que permitieron establecer tendencias de composición que distinguen a las asociaciones compuestas por gramíneas cultivadas y silvestres/ruderales.

En el Valle de Santa María, el único antecedente directo de investigación corresponde a Lanzelotti (2012), quien analizó los conjuntos fitolíticos procedentes de un sondeo efectuado en una terraza agrícola del periodo de Desarrollos Regionales en la localidad de Caspinchango (Provincia de Catamarca). Mediante este análisis identificó dos zonas en el perfil. La Zona I o inferior, dominada por elementos pooides-festucoides, estipoides y danthonioides, evidenció una vegetación herbácea de características climáticas o microclimáticas frías o condiciones de crecimiento adversas. La Zona II o superior del perfil, con elementos panicoides, chloridoideos, como asî también bambusoideos y arecoideos se vincula con una vegetación de características más cálidas y menor disponibilidad hídrica. La autora propuso que la presencia de diatomeas provendría del agua utilizada para regar estos surcos. Finalmente señala que en las unidades superiores se encuentran fitolitos que, sumado a sus abundancias, permiten proponer que las maideas (y tal vez Zea mays) habrían sido cultivadas en el sector.

Si bien existen algunos antecedentes de análisis fitolíticos en el Valle Calchaquí (Williams et al. 2010), estos son escasos y de limitado alcance, no permitiendo generar un marco interpretativo amplio de la temática. Tampoco realizan aportes vinculados a las adecuaciones ambientales de las zonas agrarias ni las interpretaciones están relacionadas con las reconstrucciones paleoambientales existentes en la zona (Peña Monné et al. 2015; Sampietro Vattuone et al. 2018).

En este contexto, el objetivo de este trabajo es realizar la caracterización de las asociaciones de microrrestos vegetales recuperados de perfiles estratigráficos localizados en terrazas de cultivo y un sector marginal al área de ocupación de la localidad de Yasyamayo (Valle de Santa María, Tucumán, Argentina), pertenecientes al periodo de Desarrollos Regionales (ca. 1000-600 AP). A partir de ellos interpretar los cambios paleombientales y antrópicos ocurridos en ese sitio. La información producida en esta investigación es novedosa, ya que no existen trabajos previos similares en la porción tucumana de los valles Calchaquíes. 


\section{Área de Estudio}

El sitio arqueológico Yasyamayo se encuentra ubicado en el piedemonte occidental de Cumbres Calchaquíes (Sampietro y Neder 2011), en la porción tucumana del Valle de Santa María en un nivel intermedio del glacis pleistoceno producto de la acumulación de flujos de detritos de alta competencia con matriz limosa y bloques de tamaño diverso posteriormente glaciplanados (Figura 1) (Sampietro Vattuone et al. 2016). Las precipitaciones medias anuales alcanzan alrededor de los $200 \mathrm{~mm}$, y los valores de evapotranspiración $700 \mathrm{~mm}$ anuales, lo cual sumado al alto déficit hídrico, de alrededor de $500 \mathrm{~mm}$, hace que la agricultura sin irrigación sea imposible en la actualidad (Pietragalla y Corso 2008).

Los suelos dominantes son Entisoles con escaso o nulo desarrollo de horizontes pedogenéticos cuyas propiedades derivan del material parental. Se trata de suelos comunes en climas extremos. En este caso, dentro del orden, es posible identificar en la zona Torriortentes típicos cuyo régimen de humedad es Tórrico, y en donde en el mejor de los casos, es posible reconocer una secuencia de horizontes A-C. Su profundidad es variable y están compuestos de materiales texturalmente diversos con acumulación de material grueso (Sayago et al. 1998).

En este sitio se registra la existencia de construcciones agrícolas de tres tipos: terrazas, despedres y unidades circulares aisladas. Las terrazas son muros simples de hasta $60 \mathrm{~cm}$ de alto desde su base, las paredes sobresalen unos $30 \mathrm{~cm}$ por encima del nivel del suelo aguas arriba y se encuentran asentadas $10 \mathrm{~cm}$ por bajo de la superficie de cultivo pasada, sin cimentaciones de ningún tipo (SampietroVattuone et al. 2016). Los despedres son montículos artificiales constituidos por cantos rodados formando acumulaciones producto de limpieza de las superficies agrícolas. Otro tipo de construcción son las unidades circulares aisladas, cuya funcionalidad no resulta clara.

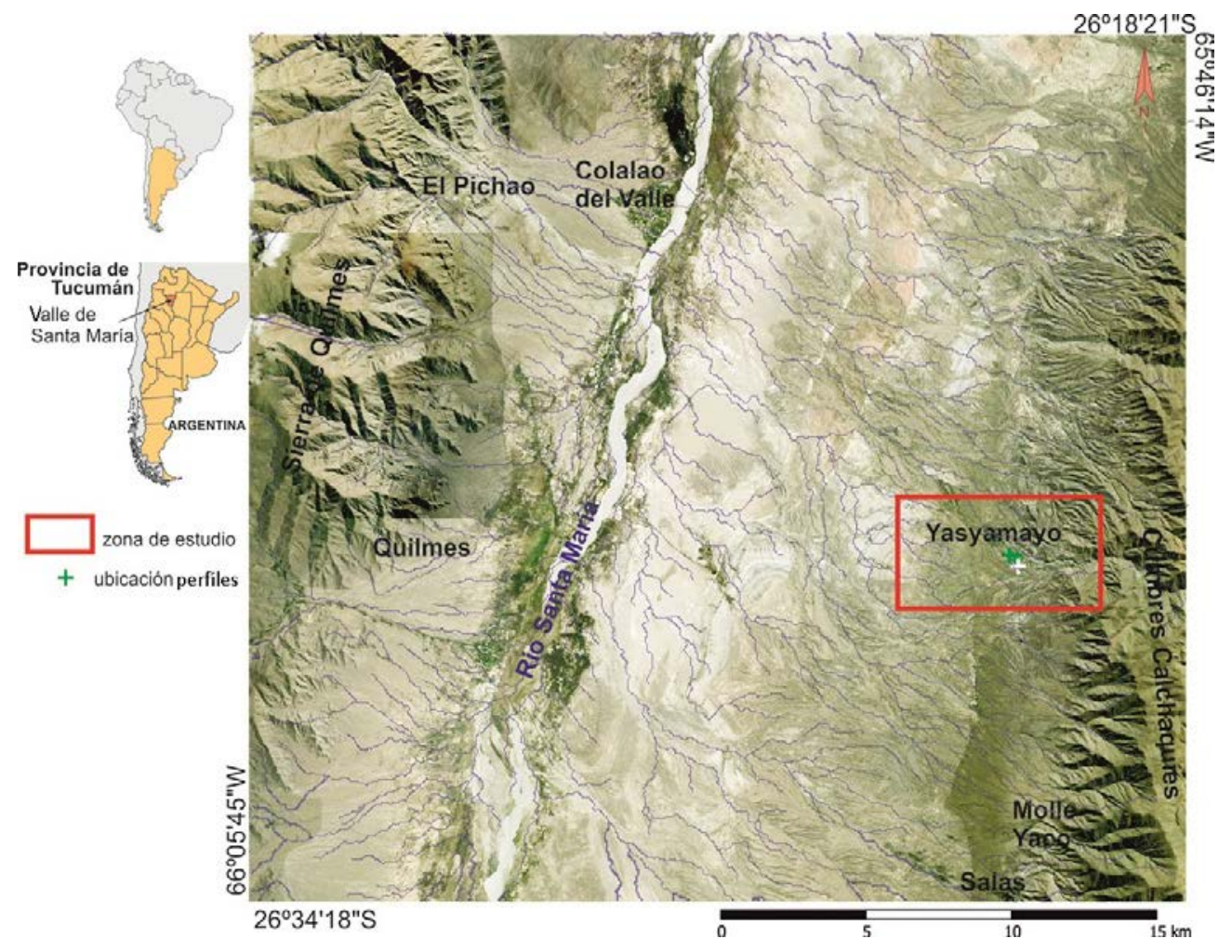

Figura 1. Ubicación geográfica del área de estudio. Yasyamayo (Dpto. Tafí del Valle, Tucumán-Argentina). Study area location. Yasyamayo (department of Tafí del Valle Tucumán-Argentina). 
Las secuencias estratigráficas presentan una textura desde arcillo limosa a limosa. En los perfiles Y-P4 e Y-P5 la textura cambia en la superficie de cultivo respecto de las otras capas, en el primer caso se hace más fina (franco arcillo limosa), y en el segundo caso un poco más gruesa (franco limosa). Por otro lado, el color cambia acompañando al cambio de textura en las capas de los perfiles consignados previamente (Y-P4: 2,5Y 7/1 e Y-P5: 2,5Y 4/2). Los perfiles agrícolas Y-P3 e Y-P4 tienen porcentajes de materia orgánica rica en la capa 1 o superficial producto del aporte de plantas y animales actuales, mientras que en el perfil agrícola Y-P5 el porcentaje de materia orgánica es extremadamente rico en la superficie de cultivo prehispánica (capa 2). En cuanto a los valores de $\mathrm{pH}$, todos los perfiles analizados son más básicos a mayor profundidad. En todos los perfiles antrópicos analizados la superficie de ocupación se diferencia según los indicadores físicos y químicos considerados. En general, este nivel se encuentra entre los 10 y $40 \mathrm{~cm}$ de profundidad, enterrado bajo una o dos horizontes de suelo (Roldan 2012) (Figura 2).

Los análisis de suelo realizados indican que los perfiles naturales son más arenosos y tienen menor concentración de nutrientes biodisponibles que los de las zonas agrícolas del sitio, donde las texturas más finas favorecieron la retención de micronutrientes y su biodisponibilidad aún en el caso de que el uso agrario haya disminuido la cantidad porcentual de materia orgánica (Sampietro Vattuone et al. 2013; 2016).

De acuerdo a las características estilísticas del material cerámico superficial encontrado se estima que los asentamientos arqueológicos pertenecen al periodo de Desarrollos Regionales (ca. 1000-600 AP) (Sampietro Vattuone et al. 2012).

El periodo Tardío o de Desarrollos Regionales (ca. 1000-600 AP) está caracterizado por: (1) la aparición de sociedades de organización política más compleja, dentro de un modelo de señoríos con el dominio territorial de uno o más valles; (2) el surgimiento de la especialización artesanal; (3) los múltiples contactos interétnicos motivados por la trashumancia; (4) el autoabastecimiento, sostenido por una economía productiva y el manejo de diversos pisos ecológicos; (5) concentración habitacional e incremento demográfico. Hay una tendencia hacia la urbanización, los poblados son de tipo concentrado con habitaciones contiguas, calles, recintos o espacios públicos abiertos, sectores de molienda de granos y de otras actividades domésticas y lugares de basurero, indicando un planeamiento en el uso del espacio. Algunos poblados se ubican sobre lomas altas en situación estratégica, rodeados por murallas defensivas. Se construyeron grandes obras comunitarias de producción agrícola tales como sistemas de andenerías, represas y canales. Adquirió un gran desarrollo la textilería en lana, el pastoreo de auquénidos y la metalurgia en bronce (Caggiano y Sempé 1994; Maldonado 2016).

\section{Vegetación actual}

El paisaje es de tipo semiárido, caracterizado por cardones, árboles y arbustos bajos, espinosos y adaptados al desierto (Cabrera 1976). Fitogeográficamente, la zona comprende cuatro regiones: Monte, Prepuna, Puna y Altoandina (Cabrera 1971). Esta diversidad de ambientes se expresa en un conjunto de comunidades vegetales divididas en estratos, a medida que aumenta la altura sobre el nivel del mar. Entre los 1.800 y 2.400 $\mathrm{msm}$ se encuentra el bosque intermontano de galería y la estepa arbustiva del monte que ocupan el 60\% de la superficie total del valle. Entre los 2.400 y los 3.000 msm están las comunidades graminosas y estepa arbustiva con cactáceas y bromeliáceas en cojín. A los $3.700 \mathrm{msm}$ se desarrollan pajonales, y por último, a los $4.600 \mathrm{msm}$ se extienden comunidades graminosas altoandinas (Escudero Martínez 1991; Perea 1991).

El área de Yasyamayo se encuentra cubierta en un $50 \%$ por bosquecitos de quebrada (Acacia visco, Salix humboldtiana y Schinus molle) distribuidos de manera agregada. En el área de estudio se encuentra una estepa arbustiva con representantes de la familia Cactaceae como Trichocereus atacamensi, Airampoa ayrampo, Tunilla tilcarensis, Parodia maassii, Parodii stuemeri, representantes de la familia Fabaceae como Prosopis ferox, Gochnatia glutinosa, Senna crassiramea, Aphyllocladus spartioides, Cercidium andicola y Zuccagnia punctata, entre otros (Oyarzabal et al. 2018). También numerosas especies de Poaceae como Digitaria californica, Munroa argentina, Jarava leptostachya, Jarava media y Eragrostis andicola (Aagesen et al. 2009). La familia Solanaceae se encuentra en esta área de estudio y forma la estepa de Fabiana densa de manera conjunta con representantes de la familia Asteraceae como Baccharis boliviensis (Puna). La vegetación zonal predominante consiste en una estepa de caméfitas y hemicriptófitas herbáceas con una composición muy heterogénea que depende de la latitud, la altitud, la exposición, el nivel de humedad y el tipo de suelo. Existe también una estepa graminosa muy abierta, de hasta $40 \mathrm{~cm}$ de altura, representada por una asociación de diferentes especies de la familia Poaceae que incluye a Festuca orthophylla, F. chrysophylla, Poa gymnantha, Stipa speciosa y Pappostipa vaginata, con varias especies cespitosas acompañantes como Pappostipa frigida, Nassella mucronata, Deyeuxia cabrerae, etc. (Cabrera 1976; Morello et al. 2012; Wingenroth y Suárez 1984).

\section{Materiales y Métodos}

En primer lugar, se realizó la prospección de la localidad de Yasyamayo para identificar sectores de explotación agraria. Se excavaron cuatro sondeos en la zona media del glacis nivel 2: un perfil testigo fuera del sector agrario y tres antropizados en el centro de 


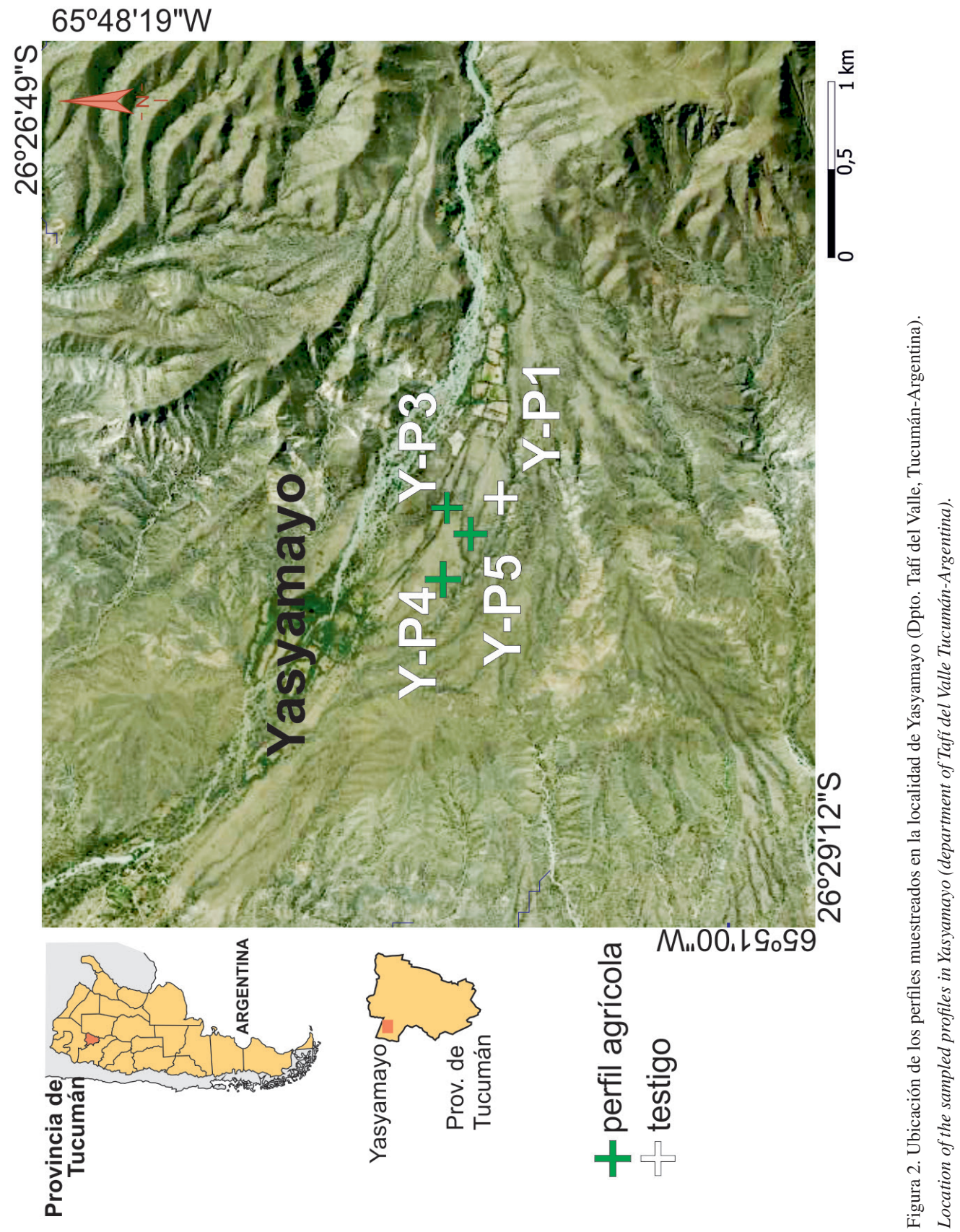


estructuras agrícolas. El perfil testigo con referencia Y-P1 (Yasyamayo, perfil 1) (26 27' 55" S, 65 50' 04'O O $2233 \mathrm{msm}$ ), de $60 \mathrm{~cm}$ de potencia, se excavó en un sector no antropizado de la misma unidad geomorfológica para obtener un patrón natural comparativo. Los perfiles antropizados son: Y-P3 (Yasyamayo, perfil 3) (26 28' 02,96”' S, 65 49' 55,57' O; $2.269 \mathrm{msm}$ ) con una potencia de $50 \mathrm{~cm}$; Y-P4 (Yasyamayo, perfil 4) con una potencia de $64 \mathrm{~cm}\left(26^{\circ} 28^{\prime} 0,179^{\prime \prime} \mathrm{S}, 65^{\circ} 50^{\prime}\right.$ 06,18" O; $2.243 \mathrm{msm}$ ); Y-P5 (Yasyamayo, perfil 5) (26 $28^{\prime} 06,15 \mathrm{~S}, 65^{\circ} 50^{\prime}$ 01,07' O; $2.255 \mathrm{msm}$ ) con $40 \mathrm{~cm}$ de potencia (Figura 3). En cada perfil las capas fueron numeradas correlativamente de arriba hacia abajo y se extrajeron muestras sedimentarias cada $10 \mathrm{~cm}$ aproximadamente.

En total se analizaron 21 muestras, en primera instancia se realizó un tratamiento preliminar, para eliminar sustancias aglomerantes de los elementos micropaleontológicos para facilitar su extracción, consistente en la eliminación de sales solubles mediante lavado con agua destilada, de carbonatos con ácido clorhídrico $1 \mathrm{~N}$, de materia orgánica con agua oxigenada al $30 \%$ en volumen y de barnices y/o cementos con ácido clorhídrico $1 \mathrm{~N}$ en caliente (Zucol et al. 2010). Después de una neutralización final del material remanente o "muestra limpia" se procedió a la desagregación química con hexametafosfato de sodio. La muestra limpia se separó granulométricamente en tres fracciones sobre las que se centraron los estudios fitolíticos. Las fracciones gruesa (mayor a $250 \mu \mathrm{m}$ de diámetro) y media (entre 53 y $250 \mu \mathrm{m}$ de diámetro) se separaron por tamizado en húmedo, mientras que la fracción fina (entre 4 y $53 \mu \mathrm{m}$ de diámetro) se separó por sifonado a partir de la suspensión de la muestra dispersada en agua destilada. Las fracciones media y fina se sometieron a una separación densimétrica con solución de politungstato de sodio (densidad de 2.345 $\mathrm{g} / \mathrm{cm}^{3}$ ) por la técnica de centrifugación (Bertoldi de Pomar 1976) extrayendo de esta manera el material liviano (incluyendo los fitolitos) presente en cada fracción. Este material concentrado se montó para su observación microscópica en preparados permanentes con bálsamo de Canadá.

Los fitolitos fueron diferenciados según clasificaciones morfológicas modificadas de acuerdo a Twiss et al. (1969), Bertoldi de Pomar (1971), Twiss (1992), Kondo et al. (1994), Zucol (1996) y los descriptores propuestos por ICPNWG (2005). La cuantificación se hizo sobre el recuento de 400 fitolitos aproximadamente por muestra y se realizaron diagramas fitolíticos utilizando el software POLPAL (Nalepka y Walanus 2003; Walanus y Nalepka 2002). Para la caracterización de los morfotipos se utilizó el esquema clasificatorio y descriptores con sus respectivos acrónimos propuestos por Patterer et al. (2011).
A partir de los recuentos se obtuvo una planilla en la cual se destacan las abundancias relativas de cada morfotipo, luego esta información es recopilada por perfil para conformar una matriz básica de datos con las muestras, con el fin de comparar y establecer la relación entre perfiles (Apéndice 1). Posteriormente estos grupos fueron analizados mediante la aplicación de un análisis multivariado (análisis de correspondencia) que permitió comparar los distintos perfiles con la intención de establecer vinculaciones entre las distintas asociaciones de los perfiles del sitio. Para la implementación de los análisis estadísticos multivariados se utilizó el programa PAST (Paleontological Statistics; Hammer et al. 2001, 2007), y particularmente el análisis de correspondencia (AMC).

Las observaciones y fotografías microscópicas fueron realizadas con un microscopio Nikon Eclipse E200 con montaje de cámara digital Nikon Coolpix S4.

\section{Resultados}

\section{Caracterización y distribución fitolítica en los perfiles}

Todos los perfiles antropizados del sitio arqueológico Yasyamayo presentan gran abundancia de microfósiles silíceos en todas sus muestras. Los elementos no diagnósticos de especies de plantas cultivadas como los elongados, poliédricos, en forma de flabelo y los elementos aguzados resultaron más abundantes.

Las asociaciones se caracterizaron por poseer fitolitos principalmente de origen graminoide, tales como prismáticos, aguzados, flabelados, conos truncados de gran tamaño y la presencia de diatomeas (Figura 4). También existe una gran abundancia elementos bilobados, conos truncados de menor tamaño o torres y cruces (Figura 5).

\section{Yasyamayo Perfil 1 (Y-P1)}

El perfil testigo cuenta con una potencia de 60 $\mathrm{cm}$, y se caracteriza por la elevada abundancia de microrrestos silíceos en todas sus muestras. En cuanto a la distribución (Figura 6) en las muestras superficiales (C1C2) se pueden diferenciar otras formas, como elementos de conducción (Du02), halterios bilobados (Ha10 y Ha13), conos truncados (Ct07 y Ct09) y elementos poliédricos (Sx03). Las diatomeas están presentes en las capas C5 y C6 coexistentes con elementos aguzados (Ac01 y Ac02), bilobados ( $\mathrm{Ha} 01$ y Ha14), flabeliformes (Fl05 y Fl06), elongados (Mp03, Mp09 y Mp10) y conos truncados (Ct10). Las muestras de la sección inferior (C6-C5), contienen conos truncados (Ct01 y $\mathrm{Ct} 02)$ y elementos poliédricos (Sx03), mientras que 


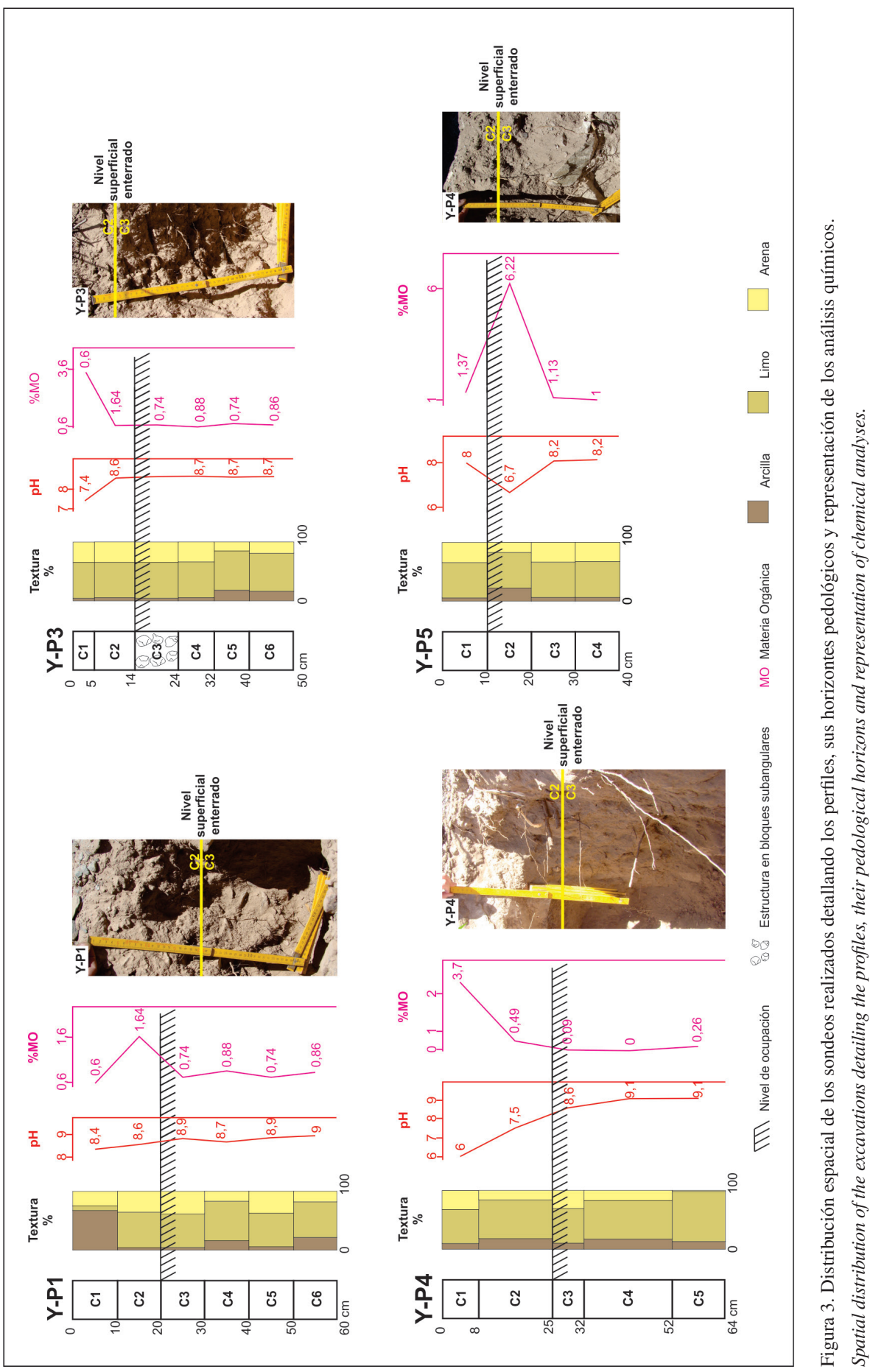




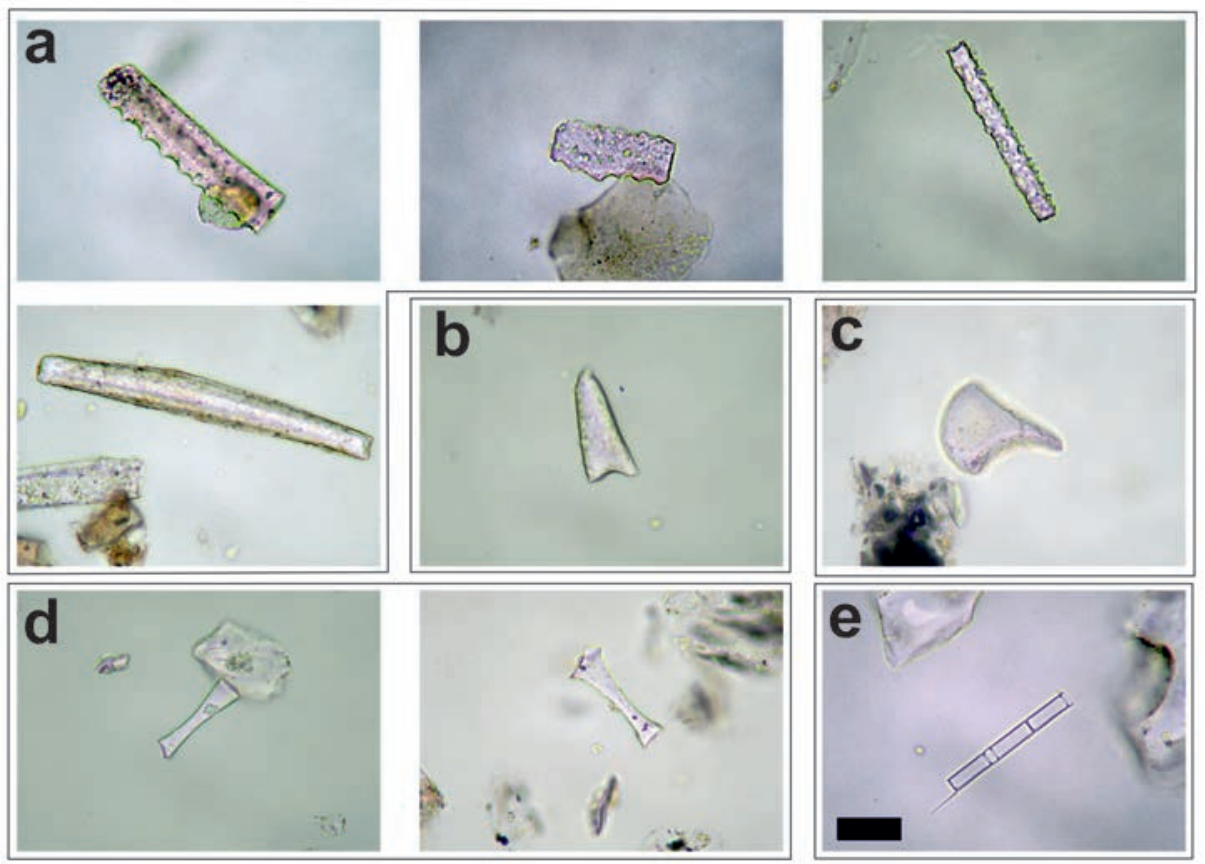

Figura 4. Elementos biosilíceos de gran tamaño, fitolitos no diagnósticos y diagnósticos observados en los perfiles estudiados: (a) fitolito prismáticos elongados de bordes denticulados y de bordes lisos (Mp); (b) elemento aguzado (Ac); (c) en forma de flabelo (Fl); (d) fitolitos en forma en forma de conos truncados (Ct); (e) diatomea. Escala gráfica en c: $20 \mu \mathrm{m}$.

Large biosiliceous elements, diagnostic and non-diagnostic phytoliths observed in the studied profiles: (a) elongated prismatic phytolith with denticulate edges and smooth edges ( $M p)$; (b) sharp element (Ac); (c) fan-shaped (Fl); d) truncated cone-shaped phytoliths $(C t) ;($ e) diatom. Linear scale $20 \mu m$.

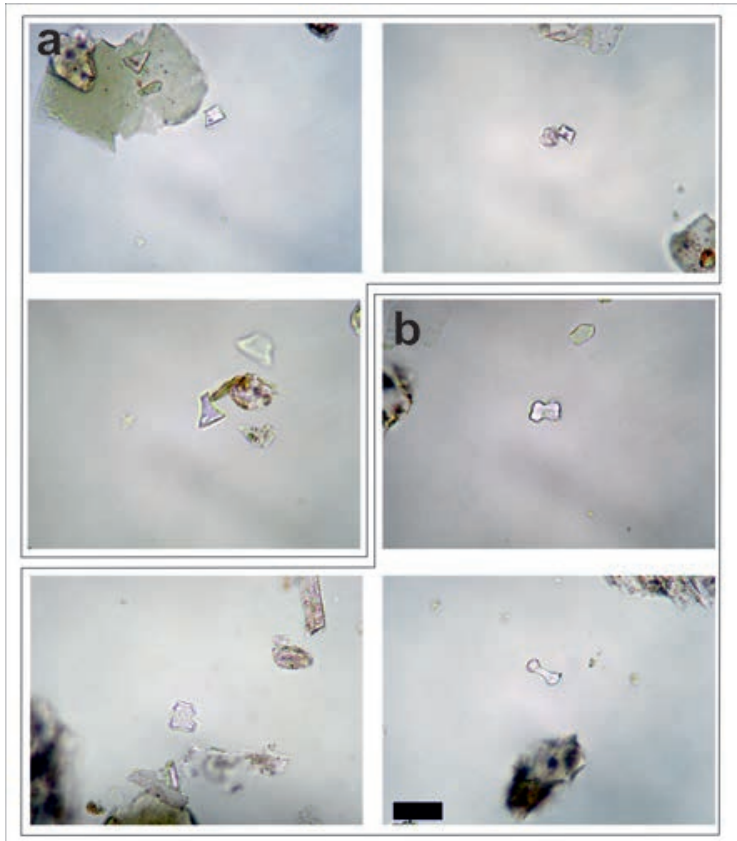

Figura 5. Fitolitos diagnósticos de menor tamaño: (a) conos truncados (Ct); (b) fitolitos bilobados halteriformes y en forma de cruz ( $\mathrm{Ha}, \mathrm{Ch})$. Escala gráfica en b: $20 \mu \mathrm{m}$.

Small size diagnostic phytoliths: (a) truncated cones (Ct); (b) Halteriform bilobate cross-shaped phytoliths. Linear scale $20 \mu \mathrm{m}$. 
la sección media del perfil (C4-C3) se caracteriza por la presencia de elementos aguzados (Ac04) y conos truncados (Ct01). En general y a lo largo de todo el perfil hay un gran número de elementos no identificados.

\section{Yasyamayo perfil 3 (Y-P3)}

El perfil antropizado Y-P3 presenta $50 \mathrm{~cm}$ de potencia con 6 capas diferenciadas. De acuerdo a Roldán (2014) la superficie de ocupación estuvo en la capa media C3. En cuanto a sus microrrestos, el perfil se caracterizó por la abundancia de elementos aguzados (Ac04, Ac06 y Ac09) dispuestos de manera uniforme y en gran número, al igual que los flabelados (Fl04 y Fl05), elongados (Mp03, Mp09 y Mp10) y prismáticos afines a gramíneas.

El análisis de cluster permite dividir el perfil entre la sección inferior (Y-P3 C4-C6) correspondiente a periodos anteriores a la ocupación, y la sección superior (Y-P3 C1-C3) que incluye el periodo de ocupación humana y capas de vegetación actual, con la presencia de picos de abundancia de cruces panicoides y elementos en forma de silla de montar afines a chloridoideas. La capa
C1-C2 contiene elementos aguzados (Ac04, Ac02 y Ac06), bilobados (Ha01, Ha14 y Ha13), flabelados (F102, Fl04 y Fl05), prismáticos (Mp03, Mp09 y $\mathrm{Mp} 10$ ), conos truncados ( $\mathrm{Ct09}$ y $\mathrm{Ct10}$ ), diatomeas y elementos globulares. La sección media C3-C4, se destaca por presentar formas flabeladas (Fl03), halterios plurilobados (Ph03 y Ph04), conos truncados (Ct07), en forma de bote (Sc01) y bilobados (Ha13) (Figura 7). Se observa en este perfil una demarcada variabilidad en torno la superficie de ocupación con presencia de diatomeas en toda la sección de la capa C3. También están presentes elementos poliédricos ( $\mathrm{Sx} 03)$, conos truncados (Ct10) y halterios (Ha14). Las cruces (Ch01) se registraron en las capas $\mathrm{C} 3$ coincidentemente con la ocupación humana.

\section{Yasyamayo Perfil 4 (YP-4)}

Este perfil se caracteriza por presentar gran abundancia de formas aguzadas (Ac02 y Ac04), sillas de montar (Sm03), halterios bilobados (Ha01 y Ha14), con forma de abanico (Fl04, Fl05 y Fl06), prismáticos elongados (Mp10, Mp09 y Mp03) y conos truncados

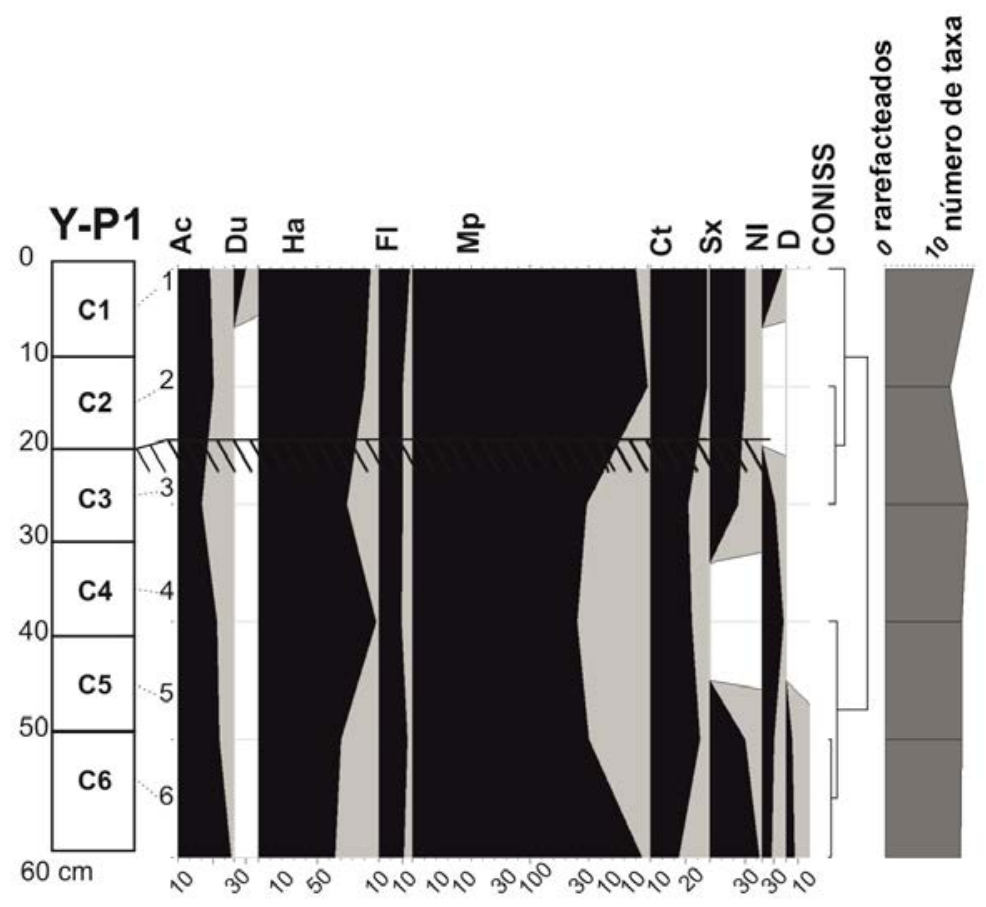

Paleosuperficie

Figura 6. Diagrama fitolítico del perfil testigo Y-P1 (en recuentos) con el análisis de variabilidad de cada muestra mediante su rarefacción y el agrupamiento de las mismas realizado mediante CONISS (Constrained Incremental Sum of Squares cluster analysis). Ac: elementos aguzados, Du: elemento de conducción, Ha: en forma de halterios, Fl: flabeliformes, Mp: fitolitos elongados de contorno liso y aserrado, Ct: conos truncados, NI: elementos no identificados, D: diatomeas.

Phytoliths diagram of Y-P1 profile (in counts) with the variability analysis of each sample through rarefaction and the grouping of them carried out by CONISS (Constrained Incremental Sum of Squares cluster analysis) by the transformation method SQRT (square root transformation). Ac: sharp element, Du: driving element, Ha: halteriform, Fl: fan-shaped, Mp: prismatic elongated phytolith with denticulate edges and smooth edges, Ct: truncated cones, NI: unidentified, D: diatoms. 


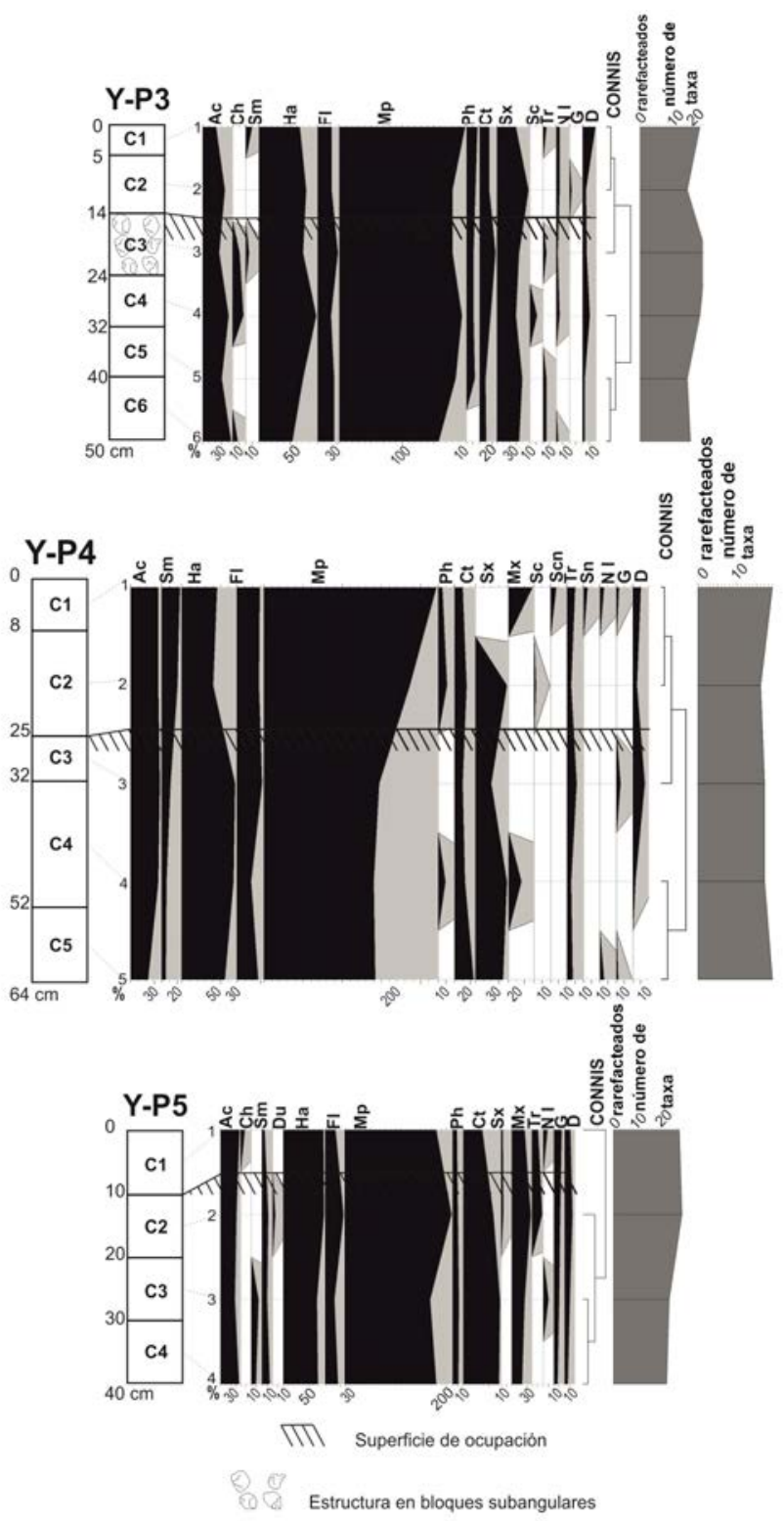

Figura 7. Diagramas fitolíticos de los perfiles antropizados (en recuento). Se observan todas las muestras con el análisis de variabilidad de cada muestra mediante su rarefacción y el análisis de la distribución de los fitolitos. Agrupamiento de las muestras realizado mediante CONISS (Constrained Incremental Sum of Squares) cluster analysis, mediante el método de transformación SQRT (square root transformation). Ac: elementos aguzados, Ha: en forma de halterios, Fl: flabeliformes, Mp: fitolitos elongados de contorno liso y aserrado, $\mathrm{Ct}$ : conos truncados, $\mathrm{Ch}$ : cruces, $\mathrm{Sm}$ : en forma de silla de montar, $\mathrm{Ph}$ : halterios plurilobados, $\mathrm{Sx}$ : poliédricos, $\mathrm{Sc}$ : en forma de bote, $\mathrm{Sn}$ : otros, $\mathrm{Mx}$ : poliédricos de menor tamaño, Tr: triangulares, NI: elementos no identificados, D: diatomeas.

Phytoliths diagram of anthropic profiles (in counts) with the variability analysis of each sample by rarefaction and the grouping of them carried out by CONISS (Constrained Incremental Sum of Squares cluster analysis) by the transformation method SQRT (square root transformation). Ac: sharp element, Ha: halteriform, Fl: fan-shaped, Mp: prismatic elongated phytolith with denticulate edges and smooth edges, Ct: truncated cones, Ch: crosses, Sm: saddle-shaped, Ph: plurilobed halter, Sx: polyhedral, Sc: boat-shaped, Sn: others, Mx: smaller size polyhedral, Tr: triangular, NI: unidentified, D: diatoms. 
largos y cortos (Ct10). La sección superficial (C2-C1) presenta gran número de elementos en forma de bote ( $\mathrm{Sc02}$ y Sn06) y en forma de silla de montar (Sm02) mientras que las muestras de la sección media (C3-C2) presentan formas bilobadas (Ha13) y flabelados (F107) coincidentes con la capa donde se encuentra el registro de la superficie de ocupación según Roldán (2014).

La sección inferior C5-C4 presenta morfotipos bilobados (Ha03), flabelados (Fl03), halterios plurilobados (Ph04), conos truncados cortos (Ct09) y poliédricos $(\mathrm{Mx} 03)$. Las diatomeas se encuentran distribuidas en todo el perfil, a excepción de la capa C5 (Figura 7).

\section{Yasyamayo Perfil 5 (Y-P5)}

El perfil antropizado Y-P5 presenta $40 \mathrm{~cm}$ de potencia, con cuatro capas: $\mathrm{C} 1, \mathrm{C} 2, \mathrm{C} 3$ y $\mathrm{C} 4$. Se puede diferenciar en este perfil, al igual que en los anteriores ya descritos, una sección inferior con las capas C4-C3 y una sección superior que incluye a las capas C2-C1. En su totalidad el perfil presenta elementos aguzados ( $\mathrm{Ac01}, \mathrm{Ac02}$, Ac04 y Ac07), en forma de silla de montar (Sm03), halterios bilobados (Ha01, Ha08 y Ha13), formas flabeladas (Fl04, Fl05, Fl06 y Fl07), elongadas (Mp03, Mp09 y Mp10), fitolitos plurilobados (Ph04), en conos truncados (Ct05, Ct07, Ct09 y Ct10), elementos poliédricos (Sx03), formas globulares y diatomeas. En la sección inferior (C4-C3) se observan elementos en forma de silla de montar (Sm03), bilobados (Ha09), formas flabeladas y conos truncados (Ct05). Además, en estas capas se encuentran otros morfotipos tales como elementos de conducción (Du01), silla de montar (Sm05), y halterios bilobados (Ha14). La sección superior presenta además elementos en forma de cruz (Ch01), flabelos (Fl03 y Fl07), conos truncados (del tipo Ct01), elementos poliédricos ( $\mathrm{Mx} 03$ ) y triangulares (Tr01). La capa $\mathrm{C} 2$ es la que posee rasgos que permiten inferir la superficie de ocupación humana (Figura 7).

Con el objetivo de sintetizar los resultados obtenidos se utilizó un análisis de correspondencia de las abundancias fitolíticas de las asociaciones (Figura 8). En los tres perfiles antropizados, a diferencia del perfil testigo, se observan las siguientes secciones: una inferior que se correspondería con la actividad sedimentaria previa a la ocupación humana; una sección media que coincide con la etapa de estabilidad y manejo agrícola del suelo y una sección superior asociada a los depósitos recientes post ocupacionales y al desarrollo de la vegetación actual. El análisis multivariado comparativo de las asociaciones fitolíticas presentes en las tres estructuras de cultivo explica una variación de tan solo el $20 \%$ entre el eje 1 y el eje 2 . Dicho análisis permite establecer que hay una marcada correspondencia de las muestras del Y-P3 y su vinculación con las muestras C2,
C3 y C5 del Y-P4, como así también, de las muestras $\mathrm{C} 2$ y $\mathrm{C} 3$ del Y-P5. Es decir que, las muestras medias de cada perfil se encuentran asociadas entre sí de manera coincidente con la superficie de ocupación humana en dichas secciones.

La asociación fitolitica de la sección inferior de todos los perfiles indica que la vegetación antes del desarrollo de las ocupaciones humanas fue dominada por elementos pooides y danthonioides característicos de plantas herbáceas típicas de zonas climáticas frías y podría vincularse además con los propios procesos de edificación de esa época (anterior al 1000 AP). Los fitolitos correspondientes a la sección media de todos los perfiles estratigráficos analizados como por ejemplo los morfotipos de gramíneas (panicoides, festucoides y chloridoides) indican condiciones áridas y semiáridas, con algunos rasgos que señalan recuperación de la vegetación. Por último, la sección superior evidencia la presencia de una vegetación similar a la actual reflejada por la presencia de fitolitos de gramíneas festucoides, chloridoides y panicoides de características climáticas cálidas con menor requerimiento de agua que lo reflejado en la sección media. Esta sección se encuentra más vinculada a florísticamente a la sección inferior.

\section{Discusión}

A partir de esta investigación se ha podido obtener evidencia sobre los tiempos anteriores, contemporáneos y posteriores a la ocupación humana reflejados en cambios en la vegetación a partir de evidencias fitolíticas, e inferir a partir de ello actividades antrópicas y condiciones paleoambientales en la porción occidental de Cumbres Calchaquíes.

En el perfil testigo, representativo de las condiciones naturales de evolución del paisaje, se puede observar en la rarefacción de los microrrestos una escasa variabilidad de fitolitos y mayor homogeneidad en toda su sección. Los conos truncados (Ct01, Ct02, $\mathrm{Ct09}, \mathrm{Ct05}, \mathrm{Ct07}$ y Ct10) se encuentran dispuestos a lo largo de todo el perfil, al igual que otras formas como las aguzadas, flabeladas, elongadas y prismáticas afines a gramíneas. Esto da pautas de que en este perfil las asociaciones vegetales se mantuvieron relativamente estables desde tiempos previos a la ocupación hasta la actualidad.

Los perfiles antropizados comparten la presencia de morfotipos semejantes entre sí, entre ellos se encuentran elementos graminoides, principalmente fitolitos en forma de conos truncados de afinidad danthonioideae, en forma de silla de montar de afinidad chloridoideae, en forma de pesas de gimnasia de afinidad panicoideae y en forma de halterios aquillados y redondeados de afinidad pooideae. Todas estas formas constituyen subfamilias dentro de la familia Poaceae, 


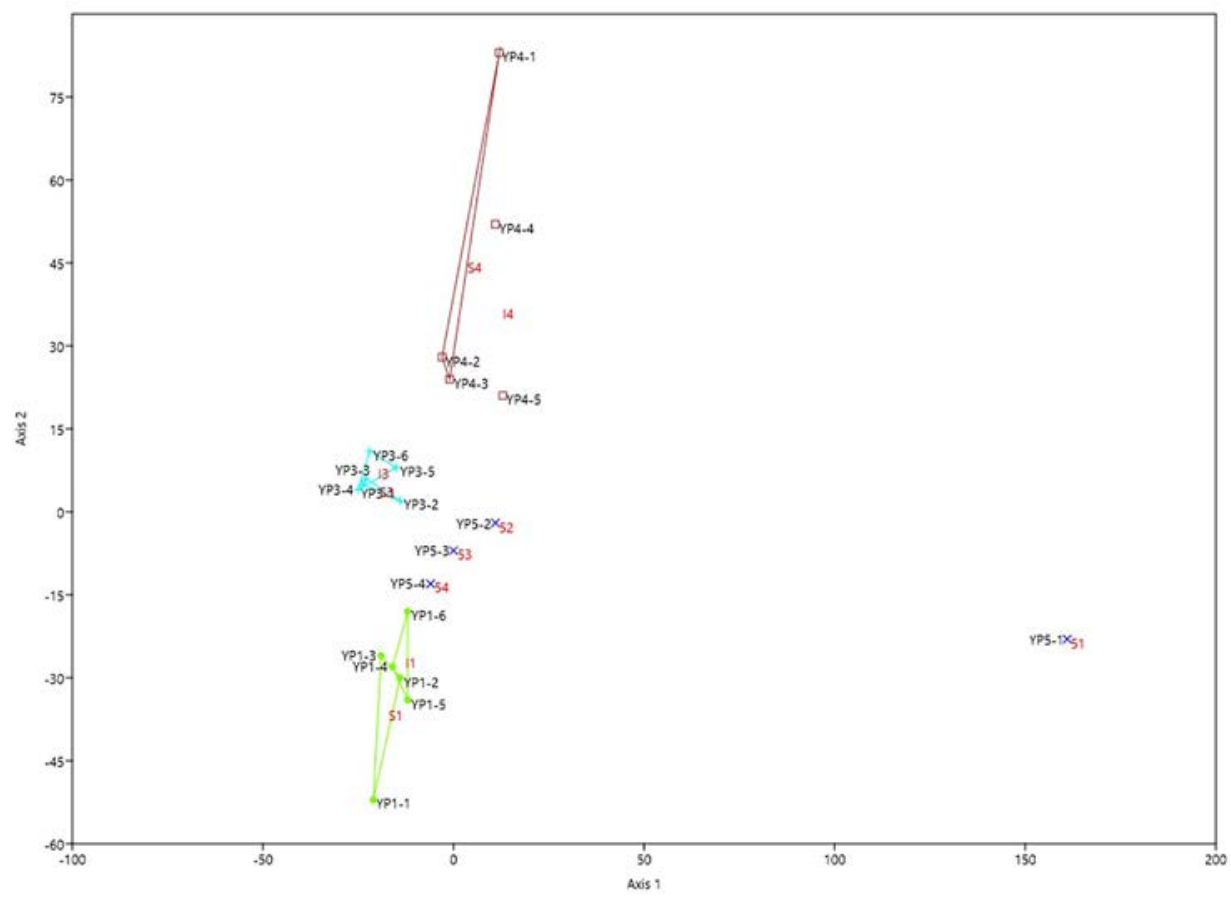

Figura 8. Análisis de correspondencia de las asociaciones fitolíticas de los perfiles Y-P3, Y-P4 e Y-P5. Porcentaje de variabilidad de los dos primeros ejes $20 \%$.

Correspondence analysis of -P3, Y-P4, and Y-P5. Percentage of variability of two axes: $20 \%$.

y se desarrollan en ambientes templados, áridos y semiáridos. Estas formas se observan principalmente en coincidencia con las capas correspondientes a la superficie de ocupación humana del periodo de Desarrollo Regionales, situada entre los 10 y $40 \mathrm{~cm}$ de profundidad e identificada previamente por Roldán (2014) en base a los análisis de indicadores físicos y geoquímicos (diferencias de estructuras pedológicas, textura, color y $\mathrm{pH}$, concentraciones elevadas de fósforo orgánico, la calidad de la materia orgánica, diferencias en las concentraciones de calcio y micronutrientes).

En ninguno de los perfiles analizados se hallaron restos arqueológicos muebles, lo que es coherente con la funcionalidad agrícola del sector y con los procesos erosivos que afectaron los sectores pedemontanos del valle (Maldonado 2016).

En los tres perfiles antrópicos se observa variabilidad de fitolitos tal como lo demuestra el incremento de la rarefacción. Los horizontes de suelo subyacentes y suprayacentes a la superficie de ocupación (capas C2, C3 y C4), están más relacionadas entre sí y presentan morfotipos que indicarían una mayor disponibilidad hídrica (conos truncados, cruces y formas bilobadas). Las diatomeas abundan a lo largo de los perfiles, y sus variaciones en correspondencia con los incrementos de las chloridoides podría considerarse un indicio de regadío en este sector durante el periodo de ocupación humana, tal como se propuso para otros campos de cultivo antiguos en el NOA (Korstanje 2010). La estructura en bloques subangulares de la capa C3 de Y-P3 indica mayor edafización por lo que en algún momento estas terrazas pudieron ser irrigadas o al menos concentrar humedad (Roldán 2012).

Además del regadío de las terrazas inferido para Yasyamayo, de acuerdo con Roldán (2012) las estrategias de manejo del suelo agrícola posiblemente incluyeron la utilización de fertilizantes orgánicos para el mantenimiento de las tierras de cultivo, considerando que la concentración de fósforo orgánico en las superficies de cultivo de las terrazas agrícolas es más elevada que las capas subyacentes. Las condiciones en las que se cultivó no eran para nada favorables, en los perfiles agrícolas Y-P3 e Y-P5 la relación de los micronutrientes es $\mathrm{Fe}>\mathrm{Cu}>\mathrm{Mn}$, donde las concentraciones de este último elemento son tan bajas que puede decirse que está prácticamente ausente.

En las capas con evidencia antrópica de las terrazas agrícolas de Yasyamayo se identificó un morfotipo de la familia de Panicoides correspondiente a maíz (Zea mays) (Pearsall 1987, 2000; Piperno 1984; Piperno y Pearsall 1998), el cual no había sido registrado hasta el momento en el área de estudio, por lo que podría suponerse que fue uno de los cultivos llevados adelante en este sector del valle durante el periodo de Desarrollos Regionales. En el Valle Calchaquí medio para el mismo periodo en estudio, Williams et al. (2010) resaltan la 
ausencia de estos silicofitolitos y solo describen muy pocos morfotipos de Poaceae, sin embargo en la zona de Ambato (Los Varela, Catamarca) se identificaron fitolitos de este cultivo junto con otros morfotipos de gramíneas (Zucol et al. 2015). No obstante ello, en Yasyamayo no se encontraron morfotipos de otras familias potencialmente relacionadas a especies cultivadas tales como Chenopodiaceae y Curcubitaceae, las cuales fueron descritas en el Valle de El Bolsón (Catamarca) por Korstanje $(2005,2009)$ y por Korstanje y Cuenya (2008).

La presencia de un morfotipo de la familia de Panicoides correspondiente a maíz (Zea mays) en las terrazas de Yasyamayo es coherente con su importancia principal dentro de los cultivos del periodo de Desarrollos Regionales, como lo evidencia su hallazgo repetido en contextos habitacionales, junto con otras especies cultivadas de las cuales no identificamos evidencias fitolíticas (Chenopodium quinoa var. quinoa, Phaseolus vulgaris var. vulgaris, Cucurbita sp.) (Cano 2011; Petrucci et al. 2018). En la economía del periodo de Desarrollos Regionales la importancia del maíz podría ser correlacionada con el aumento de productividad del mismo, la diversificación de los tipos de granos y la expansión de las áreas cultivadas (Petrucci et al. 2018).

En este punto debe señalarse que nuestras inferencias se refuerzan si se consideran las similitudes entre los perfiles (y conjuntos de microfósiles) de terrazas agrícolas del periodo de Desarrollos Regionales de Yasyamayo con otro del mismo tipo de estructura y periodo analizado por Lanzelotti (2012) para la localidad de Caspinchango. Allí también, en la zona superior del perfil correspondiente al horizonte cultivado durante los Desarrollos Regionales y al depósito posterior, se registraron elementos panicoides, chloridoideos, como así también bambusoideos y arecoideos que se vinculan con una vegetación de características más cálidas y menor disponibilidad hídrica. Similarmente, la autora propuso que la presencia de diatomeas en el horizonte cultivado debe evidenciar el regadío de estos surcos. A diferencia de Yasyamayo, en este caso se determinó un bajo nivel de fósforo en el horizonte cultivado que se explicó por la absorción de las plantas cultivadas. Finalmente, y coincidiendo con nuestro registro, en las unidades superiores del perfil se encontraron fitolitos que permiten proponer que el maíz habría sido cultivado aquí.

Por otro lado, en relación a las características paleoambientales del sector estudiado, los conjuntos fitolíticos de todos los perfiles estratigráficos analizados como por ejemplo los morfotipos de gramíneas (panicoides, festucoides y chloridoides) indican condiciones áridas y semiáridas, con algunos rasgos que señalan recuperación de la vegetación en la sección media de cada perfil. Existe una coincidencia general entre las condiciones paleoambientales inferidas al menos para el lapso de ocupación del periodo de Desarrollos Regionales (ca. 1000-600 AP) y tiempos posteriores, con las que se han propuesto para el mismo periodo en el valle. Durante los últimos 1.000 años el Valle de Santa María se caracterizó por una sucesión de fases más secas que las condiciones actuales alternantes con periodos de recuperación de la humedad (Peña Monné et al. 2015; SampietroVattuone et al. 2018). Así, en coincidencia con el periodo de Desarrollos Regionales, el ambiente fue más árido que el actual, correspondiente con la Anomalía Cálida Medieval. Luego, ya durante la Pequeña Edad de Hielo, las características ambientales oscilaron entre lapsos áridos y más húmedos, con reactivaciones dunarias durante las fases muy secas y momentos de estabilización ambiental que incluso permitieron la formación de un suelo datado en ca. $400 \mathrm{cal}$ AP en zonas del fondo de valle.Una contextualización de Yasyamayo en una mayor escala espacial permite explicar las inferencias efectuadas.

La ocupación del periodo de Desarrollos Regionales en Yasyamayo se produjo bajo un marco ambiental árido o semiárido, indicado por los conjuntos fitolíticos y por otros indicadores geoambientales para el resto del Valle de Santa María (Peña Monné et al. 2015; Sampietro Vattuone et al. 2018). En ese marco de aridez, el sistema de terrazas y el resto del sitio se construyeron en la parte media y alta del piedemonte en relación con la cuenca hídrica (o zona de cabecera) más grande del lado occidental de Cumbres Calchaquíes, lo que le aseguraba una provisión de agua más predecible, estrategia de emplazamiento que fue utilizada durante el mismo periodo en otros sectores agrarios del valle (Lanzelotti y Buzai 2015; Maldonado 2016). El sistema de terrazas de Yasyamayo es similar a los de otros sitios del valle, cuya expansión, junto con la construcción de andenes, represas, canales de regadío y depósitos para granos, fueron cambios implementados en la infraestructura agraria del periodo de Desarrollos Regionales (Tarragó, 2000) como respuesta a las condiciones de aridez. La evidencia fitolítica sugiere que en Yasyamayo se cultivó maíz, en concordancia con la especialización en su cultivo registrada a nivel del valle junto con la recolección de algarroba durante el periodo de Desarrollos Regionales (Petrucci et al. 2018). La intensificación de la producción del maíz bajo condiciones de aridez implicó el uso del riego artificial evidenciado no solo en la infraestructura de regadío sino en la presencia notable de diatomeas en la superficie de ocupación de Yasyamayo, y la pobreza de micronutrientes debió forzar el posible uso de fertilizantes evidenciados en los altos porcentajes de fósforo orgánico y materia orgánica de la superficie 
cultivada, a diferencia del Formativo donde parece no haberse usado fertilizantes (Roldán 2014). Estos cambios adaptativos en la producción agrícola identificados en Yasyamayo y en otros sitios del valle, la organización social implicada en la producción y su posterior almacenamiento (doméstica y comunal), los mecanismos de redistribución, elaboración de alimentos y bebidas (mediante la molienda, cocción, fermentación), su consumo familiar y comunal en fiestas o rituales (Petrucci y Tarragó 2015; Tarragó 2000), fueron, junto con otras actividades de subsistencia en diferentes pisos ecológicos, parte de los mecanismos que hicieron posible una organización social compleja, la cohesión y concentración habitacional y finalmente un incremento demográfico evidenciado en la expansión de las áreas habitadas bajo condiciones de aridez (Petrucci y Lema 2015).

\section{Conclusiones}

El análisis de fitolitos de interés arqueológico en el sitio Yasyamayo (Santa María - Tucumán Argentina) constituye el primer aporte al conocimiento enfocado exclusivamente en la investigación de estos microrrestos, dado que en otros trabajos solo fue utilizado como un complemento más de información. Permitió establecer que los suelos son fértiles en este tipo de microrrestos, y por tanto, constituyen una herramienta metodológica válida para la interpretación de procesos tanto antrópicos como paleoambientales, que se suman a los resultados obtenidos a partir de los análisis geoarqueológicos. Este sector del valle estuvo ocupado entre el 1000 al 1480 DC. A partir del 1000 DC, se han evidenciado para la región periodos de particular aridez, especialmente durante la ACM (Anomalía Cálida Medieval). Los fitolitos encontrados concuerdan con dichas condiciones. Los elementos afines a chloridoideae son característicos de ambientes áridos. $\mathrm{Al}$ estar dispuestos de manera alterna con las maideae, las cuales necesitan el aporte de agua, sería un indicio de que estas últimas son cultivadas.

La comparación de perfiles con y sin evidencias antrópicas permitió identificar elementos que se separan de los procesos naturales y, en este caso, el aporte de elementos panicoides como el maíz, en los niveles de ocupación, sumado a la evidencia de mayores niveles de humedad a lo largo de los perfiles, que resultan indicios que permiten estimar las estrategias empleadas por los grupos humanos de esta zona en el pasado. Las prospecciones superficiales en el área de estudio muestran la ocurrencia de estructuras de distintas características tipológicas, reafirmando el uso del espacio con fines productivos apoyando este suceso mediante el análisis macroscópico de usos de suelo, y microscópicamente mediante el análisis de microrrestos vegetales.

El desarrollo y profundización de esta línea de investigación en el futuro brindará más sustento a las interpretaciones, tanto desde la perspectiva del uso y adecuación humana del paisaje, como de los aspectos paleoecológicos evolutivos de la región.

Agradecimientos: La presente investigación fue financiada por el proyecto PIUNT G629 (Universidad Nacional de Tucumán), PIP 837 (CONICET), PICT2017-2255 (FONCYT). Agradecer a la comunidad de Amaicha del Valle en especial al Cacique Eduardo Nieva. Al Centro de Investigaciones Científicas y Transferencia de Tecnología a la Producción (CICYTTP-UADER-Provincia de Entre RíosCONICET) y al laboratorio de Biología de Agentes Bioactivos y Fitopatógenos de la Facultad de Bioquímica, Química y Farmacia (UNT). También se agradece a los evaluadores del presente artículo por los aportes y correcciones realizados.

\section{Referencias Citadas}

Aagesen, L., C.A. Szumik, F.O. Zuloaga y O. Morrone 2009. Quantitative biogeography in the South America highlands - recognizing the Altoandina, Puna and Prepuna through the study of Poaceae. Cladistics 25:295-310.

Baker, G. 1960. Fossil opal-phytoliths. Micropaleontology 6:79-85.

Bertoldi de Pomar, H. 1971. Ensayo de clasificación morfológica de los silicofitolitos. Ameghiniana 8:317-328.

Borrelli, F., M. Osterrieth y J. Marcovecchio 2008. Interrelations of vegetal cover, silicophytolith content and pedogenesis of Typical Argiudolls of the Pampean Plain, Argentina. Catena 75:146-153.

Cabrera, A.L. 1971. Fitogeografía de la República Argentina. Boletín de la Sociedad Argentina de Botánica Vol. 14. Buenos Aires.

Cabrera, A.L. 1976. Regiones fitogeográficas argentinas. En Enciclopedia Argentina de Agricultura y Jardinería. Tomo II, editado por W.F. Kugler, pp. 1-85. Fascículo 1. Editorial Acme, Buenos Aires.

Caggiano, M.A. y M.C. Sempé 1994. América, Prehistoria y Geopolítica. Ed. Tea. Buenos Aires.

Cano, S.F. 2011 Utilización de Recursos Vegetales y Subsistencia en el Valle de Santa María durante el Período de Desarrollos Regionales: Un Caso de Estudio en el Sitio El Pichao (S Tuc Tav 5). Trabajo final para optar al Título de Arqueólogo (Tesis de grado). Facultad de Ciencias Naturales e Instituto Miguel Lillo, Universidad Nacional de Tucumán, Tucumán.

Capdepont, I., L. Del Puerto y H. Inda 2005. Instrumentos de molienda: evidencias del procesamiento de recursos vegetales en la laguna de Castillos (Rocha, Uruguay). Facultad de Ciencias Sociales - UNCPBA - Argentina. Intersecciones en Antropología 6:153-166.

Escudero Martínez, M.T.I. 1991. Análisis de prioridades para la conservación en el valle del río Santa María- Sector occidental. 
Tucumán-Argentina. Seminario de la Carrera de Biología orientación Botánica, Facultad de Ciencias Naturales e Instituto Miguel Lillo, Universidad Nacional de Tucumán. Biblioteca Facultad de Ciencias Naturales e Instituto Miguel Lillo, Tucumán.

Fernández Honaine, M., M.L. Oterrieth y A.F. Zucol 2009. Plant communities and soil phytolith assemblages relationship in native grasslands from southeastern Buenos Aires province, Argentina. Catena 76 (1):89-96.

Gallego, L., A.R. Distel., R. Camina y R.M. Rodriguez Iglesias 2004. Soil phytoliths as evidence for species replacement in grazedrangelands of central Argentina. Ecography 27:1-8.

Hammer, Ø., D.A.T. Harper y P.D. Ryan 2001. Past: Paleontological Statistics Software Package for Education and Data Analysis. Palaeontologia Electronica, 4 (1), art. 4: 9, 178kb. http:// palaeoelectronica.org/2001_1/past/issue1_01.htm.

Hammer,O.,D.A.T.Harpery P.D.Ryan. 2007.PAST-Palaeontological Statistics, 1.75. 86pp. http://folk.uio.no/ohammer/past.

Iriarte, J. y E.A. Paz 2009. Phytolith analysis of selected native plants and modern soils from southeastern Uruguay and its implications for paleoenvironmental and archeological reconstruction: Quaternary International 193 (1):33-123.

IPCNWG 2005. International Code for Phytolith Nomenclature 1.0. Annals of Botany 96 (2):253-260.

Kondo, R., C. Childs e I. Atkinson 1994. Opal phytoliths of New Zealand. Maanaki Whenua Press, Lincoln.

Korstanje, M.A. 2005. La Organización del Trabajo en torno a la Producción de Alimentos en Sociedades Formativas (Provincia de Catamarca, República Argentina). Tesis Doctoral en Arqueología y Museo. Facultad de Ciencias Naturales e I.M.L. U.N.T. Tucumán.

Korstanje, M.A. 2009. Microfósiles y agricultura prehispánica: primeros resultados de un análisis multiple en el NOA. En Fitolitos: Estado Actual de su Conocimiento en América de Sur, editado por A.F Zucol, M. Osterrieth, M. Brea y N. Borrelli, pp. 249-263. Universidad Nacional de Mar del Plata, Mar del Plata.

Korstanje, M.A. 2010. Agricultural crisis in the ancient Southern Andes: Trajectories and Evidence for its Archaeological Study. Archaeological Review from Cambridge 26 (1).

Korstanje, M.A. y P. Cuenya 2008. Microfósiles y agricultura prehispánica: primeros resultados de un análisis múltiple en el Noroeste Argentino. En Fitolitos: Estado Actual de su Conocimiento en América del Sur, editado por A.F. Zucol, M. Osterrieth, M. Brea y N. Borrelli, pp. 133-147. Universidad Nacional de Mar del Plata, Mar del Plata.

Lanzelotti, S.L. 2012. Uso del Espacio y Construcción del Paisaje Agrícola en la Cuenca del Río Caspinchango, Valle de Yocavil, Provincia de Catamarca. Tomos I y II. Tesis de Doctorado, Facultad de Filosofía y Letras, Universidad de Buenos Aires, Buenos Aires.

Lanzelotti, S.L. y G. Buzai 2015. Modelos de aptitud espacial para la agricultura prehispánica y actual en el valle de Santa María, Catamarca, Argentina. Estudios Socioterritoriales. Revista de Geografía 18:139-150.

Maldonado, M.G. 2016. Cambios en la Apropiación del Espacio durante el Período Agroalfarero Prehispánico en la Vertiente Oriental de la Sierra de Quilmes (Tucumán, Argentina). Tesis de Doctorado. Universidad Nacional de La Plata. Facultad de Ciencias Naturales y Museo, La Plata.

Maldonado, M.G., M.M. Sampietro Vattuone, A.M. Blasi, C. Castiñeira Latorre, J.L. Peña Monné y M.A. Pigoni 2016.
Estratigrafía, paleoambiente y procesos de formación de sitio en El Paso durante el Holoceno Tardío (valle de Santa MaríaNoroeste Argentino). In: Sampietro Vattuone, M.M. and Peña Monné, J.L. (eds.), Geoarqueología de los Valles Calchaquíes. Laboratorio de Geoarqueología, Universidad Nacional de Tucumán, 121-144.

Maloberti, M. 2014. Prácticas campesinas en emplazamientos agrícolas formativos: el caso del Alto Juan Pablo (Belén, Catamarca). Comechingonia 18(1):139-159.

Morello, J., S. Matteucci, A. Rodríguez y M. Silva 2012. Ecorregiones y Complejos Ecosistémicos Argentinos. Orientación Gráfica Editora, Buenos Aires.

Nalepka, D. y A. Walanus 2003 Data processing in pollen analysis. Acta Paleobotanica 43 (1):125-134.

Oyarzabal, M., J. Clavijo, L. Oakley, F. Biganzoli, P. Tognetti, I. Barberis, H.M. Maturo, R. Aragón, P.I. Campanello, D. Prado, M. Oesterheld, y J.C.R. Leon 2018. Unidades de vegetación de la Argentina. Ecología Austral 28:040-063.

Patterer, N.I., E. Passeggi y A.F. Zucol 2011. Análisis fitolíticos de suelos del sudoeste de la Provincia de Entre Ríos (Argentina) como una herramienta para comprender sus procesos pedológicos. Revista mexicana de Ciencias Geológicas 18(1):32-146.

Pearsall, D.M. 1987. Evidence for prehistoric maize cultivation on raised fields at Peñon del Río, Guayas, Ecuador. PreHispanic Agricultural Fields in the Andean Region (Part 2): 279-296. BAR International Series 359.

Pearsall, D.M. 2000. Paleoethnobotany: a handbook of procedures. Academic Press. San Diego.

Pearsall, D.M. 2008. Plant Domestication and the Shift to Agriculture in the Andes. En The Handbook of South American Archaeology, editado por H. Silverman y W. Isbell pp. 105120. Springer, Nueva York.

Peña Monné, J.L., C. Sancho Marcén, M. M. Sampietro Vattuone, F. Rivelli, E.J. Rhodes, M.C. Osácar Soriano, V. Fernández Rubio y R. García-Giménez 2015. Environmental change over the last millennium recorded in the Cafayate dune field (NW Argentina). Palaeogeography, Palaeoclimatology, Palaeoecology 438:352363.

Perea, M.C. 1991. Análisis de Prioridades para la Conservación en el Valle del Río Santa María, Tucumán, Sector Oriental. Tesis de Licenciatura en Ciencias Biológicas - Orientación Botánica (ms). Facultad de Ciencias Naturales e Instituto Miguel Lillo. Universidad Nacional de Tucumán, Tucumán.

Petrucci, N.S. y M. Tarragó 2015. Restos Arqueobotánicos del Sitio Rincón Chico 1. Una aproximación a los posibles escenarios de procesamiento, uso y consumo. Comechingonia 19:67-86

Petrucci, N.S. y V.S. Lema 2015. Primeras aproximaciones a la identificación de técnicas de procesamiento en carporrestos de Zea mays L.: detección de granos hervidos en restos arqueobotánicos secos y carbonizados. Intersecciones en Antropología 17:291-302.

Petrucci, N., V.S. Lema, M.L. Pochettino, V. Palamarczuk, R. Spano y M. Tarragó 2018. From weeds to wheat: a diachronic approach to ancient biocultural diversity in the Santa María valley, northwest Argentina. Vegetation History and Archaeobotany 27:229-239.

Pietragalla, V. y M.L. Corso 2008. Proyecto de evaluación de la degradación de tierras de zonas áridas (LADA). Informe 
de avance a escala local. Secretaria de Medio Ambiente de la Nación, Argentina, ciudad.

Piperno, D.R. 1984. A comparison and differentiation of phytoliths from maize and wild grasses: use of morphological criteria. American Antiquity 49:361-83.

Piperno, D.R. y D. M. Pearsall 1998. The origins of agriculture in the Lowland Neotropics. Academic Press, San Diego.

Piperno, D.R. y J.G. Jones 2003. Paleoecological and archaeological implications of a Late Pleistocene/ Early Holocene record of vegetation and climate from the Pacific coastal plain of Panama. Quaternary Research 59:79-87.

Piperno, D.R., J.E. Moreno, J. Iriarte, I. Holst, M. Lachniet, J.G. Jones, A.J. Ranere y R. Castanzo 2007. Late Pleistocene and Holocene environmental history of the Iguala Valley, Central Balsas Watershed of Mexico. PNAS 104 (29):11874-11881.

Planella, M.T., F. Falabella, C. Belmar y L. Quiroz 2014. Huertos, chacras y sementeras. Plantas cultivadas y su participación en los desarrollos culturales de Chile central. Revista Española de Antropología Americana 44 (2):495-522.

Roldan, J. 2014. Biogeoquímica de suelos agrícolas prehispánicos en los valles Calchaquíes (Tucumán-Argentina). Tesis Doctoral. Facultad de Bioquímica, Química y Farmacia. Repositorio Institucional y Centro de Documentación Digital de la Universidad Nacional de Tucumán (RIUNT). http://www.repositorio.unt.edu. ar/handle/11327/131.

Roldan, J., M.A. Vattuone y M.M. Sampietro Vattuone 2012. Agricultura prehispánica en Yasyamayo (valle de Santa María,Tucumán, Argentina). Estudios Atacameños Arqueología y Antropología Surandinas 47:83-100.

Sampietro Vattuone, M. M. y L. Neder 2011. Quaternary Landscape Evolution and Human Occupation in Northwestern Argentina. Geological Society, London, Special Publications 352:37-47.

Sampietro Vattuone, M.M., C. Perea, M.G. Maldonado, J. Roldan y L. Neder 2012. Asentamientos formativos de la vertiente occidental de Cumbres Calchaquíes y su vinculación con las reconstrucciones paleoambientales existentes (Tucumán-Argentina). Actas $V$ Congreso Argentino de Cuaternario y Geomorfología, pp. 93102, Córdoba.

Sampietro Vattuone, M.M., Roldán, J. Maldonado, M.G. Lefebvre y M.A. Vattuone 2013. Agricultural suitability and fertility in occidental piedmont of Calchaquíes Summits (Tucumán, Argentina). Journal of Archaeological Science 52:363-375.

Sampietro Vattuone, M.M., J. Roldán, J.L. Peña Monné, M.G. Lefebvre y M.A. Vattuone 2016. Las prácticas agrícolas durante el Formativo y los Desarrollos Regionales en los sitios de Molle Yaco y Yasyamayo (valle de Santa María). En Geoarqueología de los Valles Calchaquíes, editado por M.M. Sampietro Vattuone y J.L. Peña Monné, pp. 185-211. Facultad de Ciencias Naturales e Instituto Miguel Lillo, Tucumán.

Sampietro Vattuone, M.M., J.L. Peña Monné, M.G. Maldonado, C. Sancho Marcén, W. Báez, A. Sola y A. Blasi 2018. Registro de cambios ambientales durante el Holoceno superior en depósitos fluvio-eólicos del Valle de Santa María (Noroeste Argentino). Boletín Geológico y Minero 129:647-669.
Sayago, J.M., L. Neder y M.E. Puchulu 1998. Suelos. En Geología de Tucumán, editado por M. Gianfrancisco, M.E. Puchulu, J. Durango y F. Aceñolaza, pp. 275-286. Editorial, Tucumán.

Tarragó, M.N. 2000. Chacras y pukara. Desarrollos sociales tardíos. En Nueva Historia Argentina. Los Pueblos Originarios y la Conquista, dirigido por M. Tarragó, Tomo 1, pp. 257-300. Ed. Sudamericana, Buenos Aires.

Twiss, P.C. 1992. Predicted world distribution of C3 and C4 grass phytoliths. En Phytoliths Systematics. Emerging Issues, editado por G. Rapp, Jr y S.C. Mulholland. Advances in Archaelogical and Museum Science 1:113-128.

Twiss, P.C., E. Suess y R. Smith 1969. Morphological classification of grass phytoliths. Soil Science Society of America, Proceedings 33 (1):109-115.

Walanus, A. y D. Nalepka 2002. POLPAL. Counting Pollen, Tables Storage and Diagrams Plotting. System Manual. W. Szafer Institute of Botany, Polish Academy of Sciences, Polonia.

Wilding, L.P. 1967. Radiocarbon dating of biogenetic opal. Science 184:899-901.

Williams, V., M.A. Korstanje, P. Cuenya y P. Villegas 2010. La dimensión social de la producción agrícola en un sector del Valle Calchaquí Medio. En Arqueología de la Agricultura: Casos de Estudio en la Región Andina Argentina, editado por M.A. Korstanje y M. Quesada, pp.178-207. Ediciones Magna, Buenos Aires.

Wingenroth, M. y J. Suárez 1984. Flores de Los Andes. Alta montaña de Mendoza. IANIGLA, CRICYT, Mendoza.

Zucol, A.F. 1992. Microfitolitos I: Antecedentes y terminología. Ameghiniana 29(4):353-362.

Zucol, A.F. 1996. Microfitolitos de las Poaceae argentinas: I. Microfitolitos foliares de algunas especies del género Stipa (Stipeae: Arundinoideae), de la Provincia de Entre Ríos. Darwiniana 34:151-172.

Zucol, A.F., G.G. Figueroa y M.M. Colobig 2012. Estudio de microrrestos silíceos en sistemas de aterrazamiento del primer milenio DC en el Valle de Ambato (Andes del sur), Catamarca, Argentina. Intersecciones en antropología 13 (1):163-179.

Zucol, A.F., G.G. Figueroa y M.M. Colobig 2015. Nuevos aportes para la caracterización de terrazas de cultivo del primer milenio d.C. en el valle de Ambato (Andes del Sur, Catamarca, Argentina) mediante el análisis de microrrestos. Relaciones de la Sociedad Argentina de Antropología 40 (2):425-454.

Zucol, A.F., E. Passeggi, M. Brea, N.I. Patterer, G. Fernández Pepi y M.M. Colobig 2010. Phytolith analysis for the patrokaike lake drilling proyect: Sample treatment protocols for the past microfossil manual. En $1^{a}$ Reunión Internodos del Proyecto Interdisciplinario Patagonia Austral y lerWorkshop Argentino del Proyecto Potrok Aike MaarLake Sediment Archive Drilling Project, editado por H. Corbella y N.I. Maidana, pp. 81-84. Proyecto Editorial PIPA, Buenos Aires.

Zurro, D. 2006. El análisis de fitolitos y su papel en el estudio del consumo de recursos vegetales en la prehistoria: bases para una propuesta metodológica materialista. Trabajos de Prehistoria 63 (2):35-54. 
Apéndice 1. Matriz básica de datos con los recuentos de los morfotipos fitolíticos por muestra/perfil. Ac: elemento aguzado, Du: elemento de conducción, Ha: elemento en forma de halterio, Fl: elemento en forma de flabelo, Mp: elemento elongado de contorno liso y aserrado, Ct: conos truncados, Sx: elementos poliédricos,

$\mathrm{Ch}$ : elementos en forma de cruz, Mx: poliédricos de menor tamaño, $\mathrm{Ph}$ : elemento tri y polilobados, $\mathrm{Sc}$ : elemento en forma de bote festoneado, Tr: elemento triangular, Sm: elemento en forma de silla de montar.

Basic matrix of data with morphotype phytoliths number by sample/profile.

\begin{tabular}{lrrrrrr}
\hline Nombre & YPI-1 & YP1-2 & YP1-3 & YP1-4 & YP1-5 & YP1-6 \\
\hline Ac04 & 0 & 0 & 0 & 2 & 0 & 0 \\
\hline Ac09 & 27 & 30 & 20 & 31 & 35 & 45 \\
\hline Du02 & 10 & 0 & 0 & 0 & 0 & 0 \\
\hline Ha01 & 40 & 40 & 35 & 65 & 45 & 30 \\
\hline Ha07 & 10 & 0 & 0 & 0 & 0 & 0 \\
\hline F103 & 0 & 0 & 7 & 0 & 0 & 0 \\
\hline F104 & 7 & 0 & 2 & 3 & 2 & 6 \\
\hline F105 & 6 & 10 & 7 & 15 & 17 & 10 \\
\hline F106 & 13 & 10 & 4 & 1 & 5 & 5 \\
\hline Mp03 & 40 & 35 & 25 & 30 & 40 & 40 \\
\hline Mp09 & 120 & 135 & 100 & 90 & 90 & 120 \\
\hline Mp10 & 30 & 30 & 23 & 20 & 20 & 35 \\
\hline Ct01 & 0 & 0 & 3 & 0 & 10 & 3 \\
\hline Ct05 & 0 & 0 & 0 & 5 & 0 & 0 \\
\hline Ct06 & 0 & 0 & 0 & 0 & 0 & 0 \\
\hline Ct07 & 15 & 23 & 12 & 0 & 0 & 0 \\
\hline Ct09 & 10 & 0 & 0 & 0 & 12 & 1 \\
\hline Ct10 & 23 & 25 & 17 & 30 & 20 & 20 \\
\hline Sx01 & 30 & 30 & 24 & 0 & 30 & 42 \\
\hline Ha14 & 45 & 35 & 40 & 35 & 25 & 35 \\
\hline Ha13 & 0 & 0 & 11 & 18 & 10 & 8 \\
\hline No identif. & 0 & 0 & 0 & 0 & 0 \\
\hline Globulares & 0 & 0 & 0 & 5 & 7 \\
\hline Diatomeas & 0 & 0 & 0 & 0 & 0 \\
\hline & 0 & 0 & & 0 & \\
\hline
\end{tabular}

\begin{tabular}{lrrrrrr}
\hline Nombre & YP3-1 & YP3-2 & YP3-3 & YP3-4 & YP3-5 & YP3-6 \\
\hline Ac04 & 5 & 10 & 10 & 8 & 10 & 15 \\
\hline Ac06 & 10 & 0 & 10 & 17 & 5 & 13 \\
\hline Ac09 & 6 & 25 & 6 & 16 & 15 & 17 \\
\hline Ch02 & 0 & 0 & 5 & 7 & 0 & 3 \\
\hline Ch01 & 0 & 0 & 6 & 10 & 0 & 5 \\
\hline Ha01 & 20 & 20 & 20 & 35 & 25 & 20 \\
\hline F102 & 7 & 0 & 0 & 0 & 0 & 0 \\
\hline F103 & 0 & 0 & 0 & 5 & 0 & 0 \\
\hline F104 & 5 & 10 & 10 & 7 & 8 & 9 \\
\hline F105 & 10 & 12 & 15 & 9 & 15 & 15 \\
\hline F106 & 0 & 0 & 7 & 0 & 3 & 2 \\
\hline Mp03 & 30 & 25 & 30 & 35 & 30 & 23 \\
\hline Mp09 & 120 & 135 & 118 & 135 & 115 & 105 \\
\hline Mp10 & 50 & 20 & 32 & 25 & 40 & 30 \\
\hline
\end{tabular}




\begin{tabular}{lrrrrrr}
\hline Ph04 & 7 & 3 & 4 & 11 & 13 & 0 \\
\hline $\mathrm{Ct07}$ & 0 & 0 & 0 & 8 & 0 & 0 \\
\hline $\mathrm{Ct} 09$ & 5 & 0 & 10 & 0 & 0 & 0 \\
\hline $\mathrm{Ct} 10$ & 10 & 15 & 15 & 9 & 9 & 10 \\
\hline $\mathrm{Sx} 01$ & 30 & 50 & 35 & 30 & 40 & 35 \\
\hline $\mathrm{Sc01}$ & 0 & 0 & 0 & 11 & 0 & 0 \\
\hline Tr01 & 5 & 0 & 5 & 0 & 5 & 5 \\
\hline Ha14 & 40 & 50 & 45 & 45 & 30 & 33 \\
\hline Ha13 & 5 & 5 & 5 & 11 & 15 & 0 \\
\hline No identif. & 5 & 5 & 2 & 5 & 0 & 3 \\
\hline Globulares & 0 & 3 & 0 & 0 & 0 & 0 \\
\hline Diatomeas & 20 & 5 & 6 & 11 & 3 & 3 \\
\hline
\end{tabular}

\begin{tabular}{lrrrrr}
\hline Nombre & YP4-1 & YP4-2 & YP4-3 & YP4-4 & YP4-5 \\
\hline Ac04 & 15 & 15 & 20 & 16 & 10 \\
\hline Ac09 & 20 & 20 & 17 & 19 & 12 \\
\hline Sm02 & 3 & 0 & 2 & 0 & 0 \\
\hline Sm03 & 20 & 20 & 9 & 6 & 5 \\
\hline Ha01 & 15 & 20 & 13 & 25 & 20 \\
\hline F102 & 0 & 6 & 0 & 0 & 0 \\
\hline F103 & 0 & 0 & 0 & 2 & 3 \\
\hline F104 & 10 & 9 & 7 & 5 & 20 \\
\hline F105 & 12 & 5 & 15 & 8 & 3 \\
\hline F106 & 7 & 8 & 5 & 3 & 1 \\
\hline F107 & 0 & 0 & 5 & 0 & 0 \\
\hline Mp03 & 30 & 15 & 20 & 20 & 10 \\
\hline Mp09 & 170 & 141 & 115 & 109 & 120 \\
\hline Mp10 & 20 & 30 & 12 & 11 & 12 \\
\hline Ph04 & 5 & 11 & 0 & 9 & 0 \\
\hline Ct10 & 10 & 15 & 10 & 10 & 6 \\
\hline Sx01 & 0 & 40 & 20 & 40 & 35 \\
\hline Mx03 & 30 & 0 & 0 & 16 & 0 \\
\hline Sc01 & 10 & 2 & 0 & 0 & 0 \\
\hline Tr01 & 5 & 12 & 5 & 7 \\
\hline Ha14 & 0 & 40 & 32 & 30 \\
\hline Ha13 & 0 & 15 & 0 & 0 \\
\hline Sc02 & 0 & 0 & 0 & 0 \\
\hline No identif. & 0 & 0 & 0 & 5 \\
\hline Globulares & 0 & 5 & 0 & 2 \\
\hline Diatomeas & 5 & 15 & 3 & 0 \\
\hline & & & & & \\
\hline
\end{tabular}




\begin{tabular}{|c|c|c|c|c|}
\hline Nombre & YP5-1 & YP5-2 & YP5-3 & YP5-4 \\
\hline $\mathrm{Ac0} 4$ & 7 & 3 & 20 & 13 \\
\hline Ac05 & 5 & 0 & 0 & 0 \\
\hline Ac06 & 0 & 7 & 0 & 0 \\
\hline Ac09 & 25 & 21 & 8 & 23 \\
\hline $\mathrm{Ch} 02$ & 0 & 0 & 14 & 9 \\
\hline $\mathrm{Sm02}$ & 0 & 5 & 0 & 0 \\
\hline $\mathrm{Sm03}$ & 5 & 7 & 10 & 15 \\
\hline Du04 & 0 & 5 & 0 & 0 \\
\hline Ha01 & 26 & 28 & 27 & 35 \\
\hline $\mathrm{Ha} 03$ & 8 & 3 & 0 & 0 \\
\hline $\mathrm{Ha} 09$ & 0 & 7 & 3 & 3 \\
\hline Ha11 & 3 & 3 & 0 & 0 \\
\hline $\mathrm{Fl02}$ & 0 & 0 & 0 & 5 \\
\hline Fl03 & 4 & 0 & 0 & 0 \\
\hline Fl04 & 6 & 21 & 5 & 0 \\
\hline Fl05 & 7 & 7 & 3 & 13 \\
\hline Fl06 & 3 & 6 & 0 & 7 \\
\hline Fl07 & 0 & 2 & 10 & 0 \\
\hline Mp03 & 20 & 40 & 37 & 45 \\
\hline Mp09 & 145 & 130 & 110 & 110 \\
\hline Mp10 & 15 & 40 & 21 & 25 \\
\hline $\mathrm{Ph03}$ & 3 & 0 & 3 & 4 \\
\hline $\mathrm{Ph04}$ & 5 & 10 & 9 & 7 \\
\hline $\mathrm{Ct} 01$ & 0 & 9 & 0 & 0 \\
\hline $\mathrm{Ct} 05$ & 6 & 10 & 16 & 0 \\
\hline Ct06 & 0 & 0 & 0 & 17 \\
\hline $\mathrm{Ct} 07$ & 6 & 12 & 17 & 15 \\
\hline $\mathrm{Ct} 09$ & 7 & 8 & 12 & 10 \\
\hline $\mathrm{Ct} 10$ & 18 & 20 & 27 & 28 \\
\hline $\mathrm{Sx} 01$ & 25 & 37 & 25 & 20 \\
\hline $\mathrm{Mx} 03$ & 3 & 4 & 0 & 0 \\
\hline Tr01 & 12 & 20 & 0 & 0 \\
\hline Ha14 & 0 & 25 & 30 & 25 \\
\hline $\mathrm{Ha} 12$ & 35 & 0 & 0 & 0 \\
\hline Ha13 & 7 & 14 & 6 & 5 \\
\hline No identif. & 8 & 0 & 10 & 0 \\
\hline Globulares & 15 & 12 & 10 & 7 \\
\hline Diatomeas & 11 & 15 & 10 & 6 \\
\hline
\end{tabular}


